\title{
TWO-GRAPHS, SWITCHING CLASSES AND EULER GRAPHS ARE EQUAL IN NUMBER*
}

\author{
C. L. MALlOWS AND N. J. A. SLOANE $\dagger$
}

\begin{abstract}
Seidel has shown that the number $t_{n}$ of two-graphs on $n$ nodes is equal to the number of switching classes of graphs on $n$ nodes. Robinson, and independently Liskovec, have given an explicit formula for the number $e_{n}$ of Euler graphs on $n$ nodes. It is shown here that $t_{n}=e_{n}$ for all $n$.
\end{abstract}

\section{Introduction.}

Definition 1. A two-graph [9] consists of a set $\Omega$ of nodes together with a collection of triples of elements of $\Omega$ such that each 4-element subset of $\Omega$ contains an even number of these triples.

Definition 2. Let $G$ be an (ordinary) graph with node set $\Omega$ (containing no loops or multiple edges). Let $\omega$ be a node in $\Omega$ and suppose $\omega$ is adjacent in $G$ to the (possibly empty) subset $X \subseteq \Omega$. The operation of switching at node $\omega$ ([5], [9]) transforms $G$ by deleting the edges from $\omega$ to $X$, and adding edges from $\omega$ to $\Omega-X$. In other words, take the complement of the edges incident with node $\omega$.

(i)

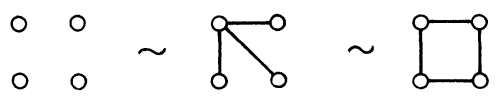

(ii)

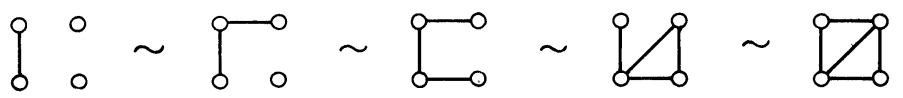

(iii)

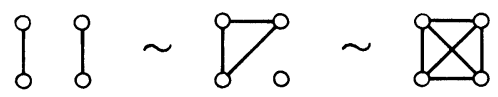

FIG. 1. The three switching classes for $n=4$

We define an equivalence relation on graphs by saying that $G^{\prime}$ is equivalent to $G$ if there exists a sequence of nodes $\omega_{1}, \omega_{2}, \cdots$ such that $G^{\prime}$ is obtained from $G$ by switching at $\omega_{1}, \omega_{2}, \cdots$. Figure 1 shows the three equivalence classes (or switching classes) of graphs with $n=4$ nodes.

Seidel [9] showed that the number $t_{n}$ of switching classes of graphs with $n$ nodes is equal to the number of two-graphs with $n$ nodes. The values of $t_{1}, \cdots, t_{7}$ were given in [5], and of $t_{8}, t_{9}$ (found by F. C. Bussemaker) in [9].

Definition 3. An Euler graph ([7], [11, p. 20]) is a graph in which every node has even degree.

Robinson [8] and independently Liskovec [6] have given formulas for the number $e_{n}$ of Euler graphs on $n$ nodes. Robinson's formula is

$$
e_{n}=\sum_{(\sigma)} \frac{2^{v(\sigma)-\lambda(\sigma)}}{\prod_{i} i^{\sigma_{i}} \sigma_{i} !},
$$

* Received by the editors April 19, 1974.

† Bell Laboratories, Murray Hill, New Jersey 07974. 
the sum being over all ordered $n$-tuples $\sigma=\left(\sigma_{1}, \cdots, \sigma_{n}\right)$ such that $n=\sum_{i} i \sigma_{i}$, where

$$
\begin{aligned}
v(\sigma)= & \sum_{i<j} \sigma_{i} \sigma_{j}(i, j)+\sum_{i} i\left(\sigma_{2 i}+\sigma_{2 i+1}+\left(\begin{array}{c}
\sigma_{i} \\
2
\end{array}\right)\right), \\
\lambda(\sigma)= & \sum_{i} \sigma_{i}-\operatorname{sgn}\left(\sum_{i} \sigma_{2 i+1}\right), \\
& \operatorname{sgn}(x)=0 \quad \text { if } x=0, \quad=1 \text { if } x>0,
\end{aligned}
$$

and $(i, j)$ is the greatest common divisor of $i$ and $j$. Both [8] and [6] give the values of $e_{1}, \cdots, e_{8}$. (The value of $e_{8}$ is given incorrectly in [4, p. 117].) Values of $e_{n}$ for $1 \leqq n \leqq 21$, obtained from (1), are given in Table 1 .

\begin{tabular}{|rl|}
\hline$n$ & $t_{n}$ \\
1 & 1 \\
2 & 1 \\
3 & 2 \\
4 & 3 \\
5 & 7 \\
6 & 16 \\
7 & 54 \\
8 & 243 \\
9 & 2,038 \\
10 & 33,120 \\
11 & $1,182,004$ \\
12 & $87,723,296$ \\
13 & $12,886,193,064$ \\
14 & $3,633,057,074,584$ \\
15 & $1,944,000,150,734,320$ \\
16 & $1,967,881,448,329,407,496$ \\
17 & $3,768,516,017,219,786,199,856$ \\
18 & $13,670,271,807,937,483,065,795,200$ \\
19 & $94,109,042,015,724,412,679,233,018,144$ \\
20 & $1,232,069,666,043,220,685,614,640,133,362,240$ \\
21 & $30,739,974,599,837,035,594,494,908,346,443,726,272$ \\
\hline
\end{tabular}

In collecting data for a supplement to [12] it was observed that the numbers $e_{n}$ and $t_{n}$ agree for $1 \leqq n \leqq 8$. It is the object of this note to prove the following.

THEOREM 1. $e_{n}=t_{n}$ for all $n$.

This result had already been established by Seidel [10] for $n$ odd. However, the result when $n$ is even seems to lie much deeper. Indeed, when $n$ is odd, it is not difficult to show that there is a unique Euler graph in each switching class, thus establishing a 1-1 correspondence between the two families. But for $n$ even, we have been unable to find such a correspondence. 
2. The labeled case. Before proving Theorem 1, we consider the corresponding problem when the nodes of the graph are labeled, say with the labels $\{1,2, \cdots, n\}$. Define

$\mathscr{G}_{n}=$ set of all labeled graphs on $n$ nodes,

$\mathscr{E}_{n}=$ set of all Euler graphs in $\mathscr{G}_{n}$,

$\tilde{T}_{n}=$ set of switching classes in $\mathscr{G}_{n}$,

$\mathscr{C}_{n}=$ set of all cocycles in $\mathscr{G}_{n}([3$, p. 38], [1, p. 13]. In the notation of $[11$, p. 28],

$\mathscr{C}_{n}=$ cutsets of the complete graph on $\{1, \cdots, n\}$.

For $A, B \in \mathscr{G}_{n}$, define $A+B \in \mathscr{G}_{n}$ to be the graph containing the edges in either $A$ or $B$ but not both. With this definition of addition, $\mathscr{G}_{n}, \mathscr{E}_{n}, \mathscr{C}_{n}$ are elementary abelian groups of type $(1,1, \cdots, 1)$ of orders $G_{n}=2^{\left(\begin{array}{l}n \\ 2\end{array}\right)}, E_{n}=2^{\left(\begin{array}{c}n-1 \\ 2\end{array}\right)}$, $C_{n}=2^{n-1}$ respectively [1], [11].

Observe that two graphs are in the same switching class if and only if their sum is a cocycle. Hence $\mathscr{T}_{n} \cong \mathscr{G}_{n} / \mathscr{C}_{n}$, and has order $T_{n}=2^{\left(\begin{array}{c}n \\ 2\end{array}\right)} / 2^{n-1}=2^{\left(\begin{array}{c}n-1 \\ 2\end{array}\right)}=E_{n}$.

It is easy to construct a $1-1$ correspondence between $\mathscr{E}_{n}$ and $\mathscr{T}_{n}$. Let $\tau$ denote the tree with edges $12,13, \cdots, 1 n$, and let the edges $i j(1<i<j)$ not in $\tau$ be called (in this section only) chords. As a basis for $\mathscr{E}_{n}$ take the fundamental circuits [11, p. 27] $\Phi_{1}, \cdots, \Phi_{d}, d=\left(\begin{array}{c}n-1 \\ 2\end{array}\right)$, each consisting of a chord and the two coterminal edges of $\tau$. Any element of $\mathscr{E}_{n}$ can be written uniquely as $\sum_{i=1}^{d} a_{i} \Phi_{i}$, $a_{i}=0$ or 1 . Each switching class contains exactly one chordal graph that has no edges of $\tau$; as a basis for these graphs we take the unichordal graphs $\psi_{1}, \cdots, \psi_{d}$ that each consist of a single chord. Then each chordal graph can be written uniquely as $\sum_{i=1}^{d} a_{i} \psi_{i}, a_{i}=0$ or 1 . Thus we have proved the following.

THEOREM 2. There is a 1-1 correspondence between the $2^{\left(\begin{array}{c}n-1 \\ 2\end{array}\right)}$ labeled Euler graphs and labeled switching classes on $n$ nodes.

Figure 2 shows the correspondence when $n=4$.

3. The unlabeled case: Proof of Theorem 1. For a subset $C \subseteq\{1, \cdots, n\}$, let $X(C)$ denote the operation of switching at all the nodes of $C$ (in any order). $X(C)$ induces a permutation (which we also denote $X(C)$ ) on the set $\mathscr{G}_{n}$ of all labeled graphs. Note that $X(C)$ and $X(\bar{C})$ are the same permutation (where $\bar{C}$ is the complement of $C$ ).

The number $t_{n}$ of switching classes is the number of equivalence classes in $\mathscr{G}_{n}$ under the combined action of all $X(C)$ and all permutations $\pi$ of the $n$ nodes. The set of all $X(C)$ and all $\pi$ generate a group of order $2^{n-1} n$ !, consisting of pairs $(\pi, C)$. By Burnside's lemma [2],

$$
t_{n}=\frac{1}{2^{n} n !} \sum_{\pi \in \mathscr{S}_{n}} \sum_{C \subseteq\{1, \cdots, n\}} f(\pi, C),
$$

where $f(\pi, C)$ is the number of graphs that are fixed under the operation of first permuting the nodes according to $\pi$ and then switching at the nodes in $C$. We proceed to calculate $f(\pi, C)$.

Suppose $\pi$ permutes the nodes in $\sigma_{i}$ cycles (called node-cycles) of length $i$, for $1 \leqq i \leqq n$. Let $\beta_{1}, \beta_{2}, \cdots, \beta_{c}$ be the (node-) cycles of $\pi$, with lengths 
$b_{1}, b_{2}, \cdots, b_{c}$ respectively $\left(c=\sum \sigma_{i}, \sum b_{j}=n\right)$, and let $\gamma\left(\beta_{i}, C\right)$ be the number of nodes of $\beta_{i}$ that are in $C$.

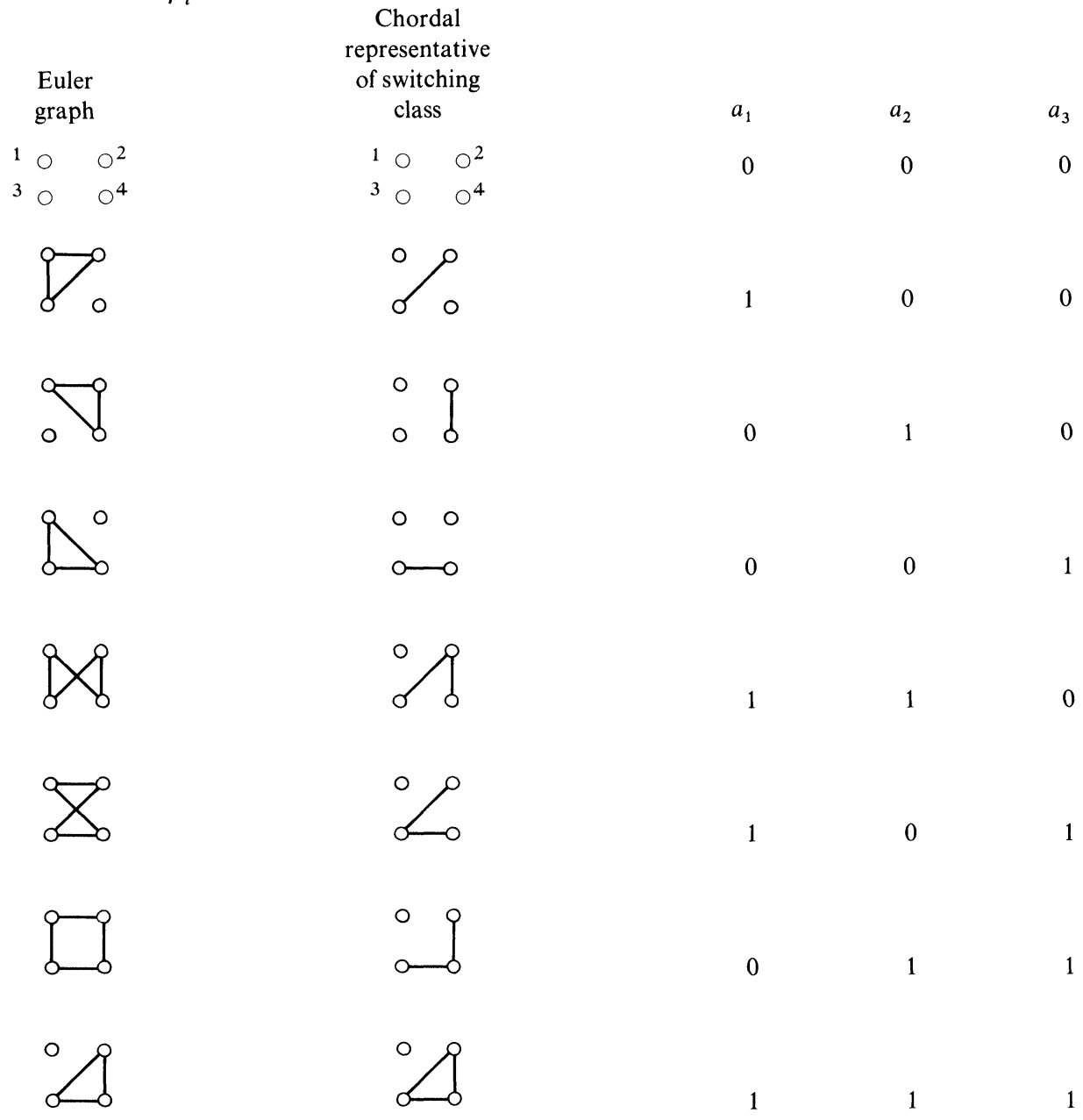

FIG. 2. 1-1 correspondence between labeled Euler graphs and labeled switching classes on four nodes

Under the action of $\pi$, the edges of the complete graph are permuted in cycles (called edge-cycles). It is shown in [8] that the total number of edge-cycles is $v(\sigma)$.

(i) If $\beta=\left(a_{1}, \cdots, a_{2 i}\right)$ is a node-cycle of length $b=2 i$, the edges $a_{\mu} a_{v}$ $(1 \leqq \mu<v \leqq 2 i)$ are permuted in $i-1$ distinct edge-cycles of length $2 i$, and one edge-cycle (the cycle of diameters) of length $i$. Then as a diameter traverses the edge-cycle of length $i$ under repeated applications of $(\pi, C)$, it is switched $\gamma(\beta, C)$ times. Thus in order for it to be possible for a graph to be fixed under $(\pi, C)$, $\gamma(\beta, C)$ must be even. An edge which is not a diameter will be switched $2 \gamma(\beta, C)$ times, which is always even. Thus if $\gamma(\beta, C)$ is even, there are $2^{i}$ ways of inserting the chords of $\beta$ so that this subgraph is fixed under $(\pi, C)$; if $\gamma(\beta, C)$ is odd, there are no such ways.

(ii) The edges joining the nodes of a node-cycle of length $2 i+1$ comprise $i$ distinct edge-cycles each of length $2 i+1$. An edge in one of these cycles is switched 
an even number of times during $2 i+1$ successive applications of $(\pi, C)$. Thus there are $2^{i}$ ways of inserting the chords of such a node-cycle, for any $C$.

(iii) Let $\beta=\left(a_{1}, \cdots, a_{b}\right), \beta^{\prime}=\left(a^{\prime}{ }_{1}, \cdots, a_{b^{\prime}}^{\prime}\right)$ be distinct node-cycles. Let $t=\left(b, b^{\prime}\right)$, and write

$$
b=r t, \quad b^{\prime}=r^{\prime} t, \quad \text { where }\left(r, r^{\prime}\right)=1 .
$$

The $b b^{\prime}$ edges $a_{\mu} a_{\mu^{\prime}}^{\prime}$ are permuted in $t$ distinct edge-cycles of length $r r^{\prime} t$ each.

As an edge traverses its edge-cycle, it is switched $p=r^{\prime} \gamma(\beta, C)+r \gamma\left(\beta^{\prime}, C\right)$ times. If both $b$ and $b^{\prime}$ are even, and an invariant subgraph is possible, both $\gamma(\beta, C)$ and $\gamma\left(\beta^{\prime}, C\right)$ must be even by (i). If $b$ is even and $b^{\prime}$ odd, $r$ and $\gamma(\beta, C)$ must be even. But if both $b$ and $b^{\prime}$ are odd, so $r, r^{\prime}$ are odd, then for $p$ to be even, $\gamma(\beta, C)$ and $\gamma\left(\beta^{\prime}, C\right)$ must both be even or both odd. Therefore if an invariant subgraph is to be possible, all node-cycles of odd length must contain an even number of points of $C$, or all must contain an odd number of points of $C$. If this holds, the number of ways of drawing an invariant subgraph of edges from $\beta$ to $\beta^{\prime}$ is $2^{t}$.

(iv) Putting all this together, the number of ways of drawing a graph that is invariant under $(\pi, C)$ is $f(\pi, C)=2^{v(\sigma)} \xi(\pi, C)$, where $\xi(\pi, C)=0$ or 1 , and $=1$ if and only if $\gamma\left(\beta_{i}, C\right)$ is even whenever $b_{i}$ is even, and $\left\{\gamma\left(\beta_{j}, C\right): b_{j}\right.$ odd $\}$ are all even or all odd together. It follows that

$$
\sum_{C} \xi(\pi, C)=2^{n-\lambda(\sigma)}
$$

Hence from (4) and (1),

$$
t_{n}=\sum_{\pi} 2^{v(\sigma)-\lambda(\sigma)}
$$

which collapses to (1), and the theorem is proved.

\section{REFERENCES}

[1] C. BERGE, Graphs and Hypergraphs, North-Holland, Amsterdam, 1973.

[2] N. G. DE Bruijn, Pólya's theory of counting, Applied Combinatorial Mathematics, E. F. Beckenbach, ed., John Wiley, N.Y., 1964, pp. 144-184.

[3] F. Harary, Graph Theory, Addison-Wesley, Reading, Mass., 1969.

[4] F. Harary and E. M. Palmer, Graphical Enumeration, Academic Press, New York, 1973.

[5] J. H. van LinT AND J. J. SeIDel, Equilateral point sets in elliptic geometry, Indag. Math., 28 (1966), pp. 335-348 = Proc. Koninkl. Nederl. Akademie van Wetenschappen, Series A, 69 (1966), pp. $335-348$.

[6] V. A. Liskovec, Enumeration of Euler graphs, Vescī Akad. Navuk BSSR Ser. Fìz.-Mat. Navuk., (1970), no. 6, pp. 38-46. (Math. Rev., 44 (1972), p. 1195 (\#6557).)

[7] R. C. READ, Euler graphs on labelled nodes, Canad. J. Math., 14 (1962), pp. 482-486.

[8] R. W. Robinson, Enumeration of Euler graphs, Proof Techniques in Graph Theory, F. Harary, ed., Academic Press, N.Y., 1969, pp. 147-153.

[9] J. J. Seidel, A survey of two-graphs, Proc. Int. Coll. Theorie Combinatorie, Acc. Naz. Lincei, Rome, 1973.

[10] - Graphs and two-graphs, Proc. 5th Southeastern Conf. on Combinatorics, Graph Theory, and Computing, Utilitas Mathematica Publishing Inc., Winnipeg, Canada, 1974.

[11] S. SeShu AND M. B. ReED, Linear Graphs and Electrical Networks, Addison-Wesley, Reading, Mass., 1961.

[12] N. J. A. Sloane, A Handbook of Integer Sequences, Academic Press, New York, 1973. 